\title{
Remyelination therapy for demyelinating disease
}

\section{Ian D. Duncan and Abigail Radcliff}

In their critique of a Review on leukodystrophies in Nature Reviews Neurology ${ }^{1}$, van der Knaap and Bugiani (Leukodystrophies - more than just diseases of myelin. Nat. Rev. Neurol. 14, $747-748(2018))^{2}$ challenge the idea that remyelination will be a useful therapeutic approach for all of these disorders and cite our paper ${ }^{3}$ as proposing this notion. They state "also, the incorrect belief that all leukodystrophies revolve around myelin implies that therapy should be focused on remyelination." We wish to clarify this issue, as this was not what we proposed. Our paper was part of a special issue on remyelination, and we were charged with discussing demyelinating diseases and identifying those disorders in which remyelination could be a therapeutic target. We reviewed practically all human and animal disorders of myelin in some detail. We went to great lengths to identify those disorders in which remyelination and its promotion might be feasible, while discussing those disorders where myelin pathology was present but evidence of significant demyelination was lacking, for example, Canavan disease, vanishing white matter disease and certain mitochondrial mutations. We stressed, as did van der Knaap and Bugiani ${ }^{2}$, that a greater understanding of the pathophysiology of the white matter disturbance in certain myelin diseases would be required before therapeutic strategies should be attempted. We did not, therefore, promote remyelination as a panacea, but recognized it as an essential process in both restoring function and protecting axons in demyelinating disease, especially multiple sclerosis.

Ian D. Duncan ${ }^{凶}$ and Abigail Radcliff Department of Medical Sciences, School of Veterinary Medicine, University of Wisconsin-Madison, Madison, WI, USA.

凶e-mail:duncani@svm.vetmed.wisc.edu https://doi.org/10.1038/s41582-020-0341-7

1. Köhler, W., Curiel, J. \& Vanderver, A. Adulthood leukodystrophies. Nat. Rev. Neurol. 14, 94-105 (2018).

2. van der Knaap, M. S. \& Bugiani, M. Leukodystrophies - more than just diseases of myelin. Nat. Rev. Neurol 14, 747-748 (2018).

3. Duncan, I. D. \& Radcliff, A. B. Inherited and acquired disorders of myelin: the underlying myelin pathology. Exp. Neurol. 283, 452-475 (2016).

Competing interest

The authors declare no competing interests. 